Published in Zheng, G., Belavy, D., Cai, Y., \& Li, S. (2019). Computational methods and clinical applications for spine imaging : 5th International Workshop and Challenge, CSI 2018, held in conjunction with MICCAI 2018, Granada, Spain, September 16, 2018, Revised selected papers. Cham: Springer.

https://doi.org/10.1007/978-3-030-13736-6

\title{
Towards a Deformable Multi-Surface Approach to Ligamentous Spine Models for Predictive Simulation- Based Scoliosis Surgery Planning
}

\author{
Michel A. Audette ${ }^{1}$, Jerome Schmid ${ }^{2}$, Craig Goodmurphy ${ }^{3}$, Michael Polanco ${ }^{1}$, \\ Sebastian Bawab ${ }^{1}$, Austin Tapp ${ }^{1}$, H. Sheldon St-Clair ${ }^{4}$. \\ ${ }^{1}$ Old Dominion University, Norfolk, VA, USA; ${ }^{2}$ School of Health Sciences - Geneva, \\ HES-SO University of Applied Sciences and Arts Western Switzerland; \\ ${ }^{3}$ Eastern Virginia Medical School \& ${ }^{4}$ Children's Hospital of The King's Daughters, Norfolk VA
}

\begin{abstract}
Scoliosis correction surgery is typically a highly invasive procedure that involves either an anterior or posterior release, which respectively entail the resection of ligaments and bone facets from the front or back of the spine, in order to make it sufficiently compliant to enable the correction of the deformity. In light of progress in other areas of surgery in minimally invasive therapies, orthopedic surgeons have begun envisioning computer simulation-assisted planning that could answer unprecedented what-if questions. This paper presents preliminary steps taken towards finite elements simulation-based surgery planning that will provide answers as to how much anterior or posterior release is truly necessary, provided we also establish the amplitude of surgical forces involved in corrective surgery. This question motivates us to pursue a medical image-based anatomical modeling pipeline that can support personalized finite elements simulation, based on models of the spine that not only feature vertebrae and intervertebral discs (IVDs), but also descriptive ligament models. This paper suggests a way of proceeding, based on the application of deformable multi-surface Simplex model to a CAD-based representation of the spine that makes explicit all spinal ligaments, along with vertebrae and IVDs. It presents a preliminary model-based segmentation study whereby Simplex meshes of CAD vertebrae are registered to the subject's corresponding vertebrae in CT data, which then drives ligament and IVD model registration by aggregation of neighboring vertebral transformations. This framework also anticipates foreseen improvements in MR imaging that could achieve better contrasts in ligamentous tissues in the future.
\end{abstract}

Keywords: Scoliosis surgery, surgery planning, finite elements simulation, spinal ligaments, minimally invasive surgery, surface meshing, mesh repair.

\section{Introduction}

\subsection{Background - Scoliosis}

Scoliosis is a medical condition in which a person's spinal axis has a three-dimensional deviation, which viewed from the rear can resemble an "S" or a "C" rather than a 
straight line. Scoliosis is defined as a spinal curvature of more than 10 degrees to the right or left in the coronal plane. Deformity may also exist in the sagittal plane. Its causes include neuromuscular problems, genetic conditions, and limb length inequality. Scoliosis is typically classified as either congenital (caused by vertebral anomalies present at birth), idiopathic (cause unknown), or secondary to a primary condition. X-rays are usually taken to assess the scoliosis curves as well as the kyphosis and lordosis, convex and concave curvatures in the sagittal plane that can also individuals with scoliosis. Instrumented scoliosis surgery was first performed in the 1960s [1], subsequent to which, device and technique modifications since then have led to improved surgical results. The goals of surgical management of adolescent idiopathic scoliosis (AIS) include maintaining coronal and sagittal alignment, producing level shoulders, correcting deformity, and saving motion segments [2]. Classification systems for AIS are useful for surgical planning and for comparing postoperative results. However, choosing optimal fusion levels remains challenging; in a study by Lenke, an average of five different proximal fusion levels and four different distal fusion levels were identified in AIS cases presented to 28 surgeons [3].

Computer simulation of scoliosis treatment can provide an efficient, risk-free means of finding the optimum among competing therapeutic approaches. The main objective of this project will be to predict the amplitude of the forces needed to correct the scoliotic spine, for example to ensure adequate fixation. However, a long-term objective is to provide the orthopedic surgery community with a predictive planning tool that enables the exploration of what-if scenarios by clinicians both as individuals and as a community, which will likely lead to greater consensus for various categories of scoliotic deformity as well as for other orthopedics cases (herniated disc, trauma, and so on) in the long term.

The proposed study describes a first instantiation of a deformable multi-surface approach to constructing a ligamentous patient-specific anatomy; it is presented as a preliminary result that will be improved upon through an on-going approach founded on multi-material surface extraction, whose objective will ultimately be to preserve shared boundaries (flush surfaces) where appropriate. The main objective of this study is to demonstrate the feasibility of using the model-image transformations undergone by vertebral surfaces of a descriptive spine model, drawn with CAD software by an anatomist, to anchor the nonrigid transformation of neighboring soft tissues (IVDs, ligaments). Moreover, these soft-tissue transformations may be further refined by medical images if adequate contrast is available, particularly as imaging techniques (MRI pulse sequences and high-field imaging) evolve to capture greater anatomical details.

\subsection{Limitations in Current Scoliosis Surgery Planning - Impetus for Minimally Invasive Approach}

For many scoliosis cases, the rigidity of the deformity cannot be overcome enough to achieve satisfactory correction, without using measures to make the spine more compliant. In these cases, a release procedure, either anterior or posterior, is used to render the spine more flexible and enable correction, albeit at the cost of a more complex and extensive procedure, as shown in figure 1. In an anterior release, as in figure 1a, inter- 


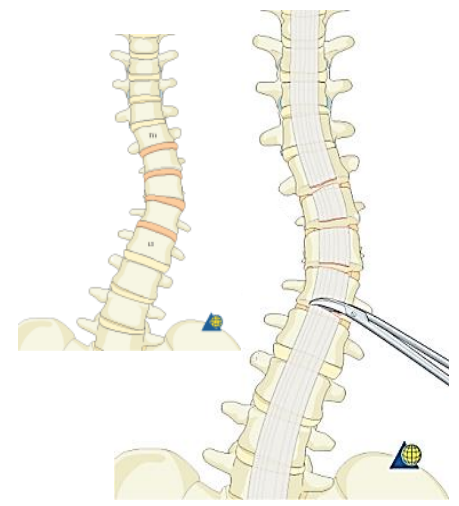

(a)

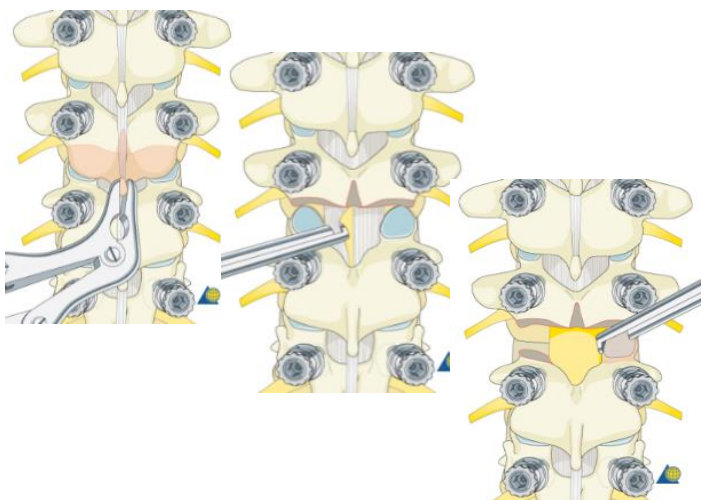

(b)

Fig. 1. (a) Anterior release: discectomy and resection of annulus of every intervertebral disk within the instrumented area: the annulus is incised from the lateral aspect of the spine, the disk is removed with curettes and rongeurs. The anterior longitudinal ligament is cut; the posterior annulus may be removed. (b) Posterior column osteotomy (left to right). First, resect the inferior aspect of the spinous process, followed by removal of the interspinous ligament with a rongeur. Second, remove 3-5 mm of inferior aspect of inferior facet joint at each level of the planned fusion with an osteotome. The ligamentum flavum is removed with a Kerrison rongeur while avoiding to penetrate deeply against the dura. Last, the rongeur is utilized to remove the superior portion of the superior articular facet. Reproduced with permission; copyright by AO Foundation, Switzerland [4].

vertebral disc (IVD) tissue is removed from the front. Furthermore, the anterior longitudinal ligament is cut at each relevant IVD. Alternately, posterior column osteotomy, of Smith-Petersen or Ponte type, involves the posterior removal of ligament and bone, as in figure $1 \mathrm{~b}$, including parts of the spinous process and facets to partially correct scoliosis. Subsequently, the surgeon inserts pedicle screws, typically in both vertebral pedicles, one of which will be used to cup a portion of a curved rod that mirrors the deformation of the spine of the patient. This curved rod has a personalized shape, which can be produced during the procedure by the surgeon himself, so as to correspond to the scoliotic curvature of the patient. Once the curved rod is inserted in all of the corresponding pedicle anchors, the surgeon imparts a 90-degree rotation to this curved rod, which effectively straightens the spine, as seen in figure 2 . Typically, this correction requires a significant amount of force, even after anterior/posterior release, and necessitates a pair of vice-grip-like surgical pliers to lock onto two points on the curved rod.

If the amplitude of corrective forces were known prior to surgery, surgical workflow would be improved, the patient would spend less time in the operating room, while limiting anterior and posterior release procedures to a minimum and generally facilitating their planning. While finite-elements-based biomechanical studies in surgery are not new, they emphasize pedicle screw insertion mechanics [5] [6]. Meanwhile, patientspecific anatomical models that account for interaction between vertebrae, bound to each other by ligaments, are generally not found in the literature or in clinical practice. As a result, existing work in surgery planning or simulation does not provide a surgeon with an estimate of the amplitude of corrective forces involved in scoliosis surgery. 

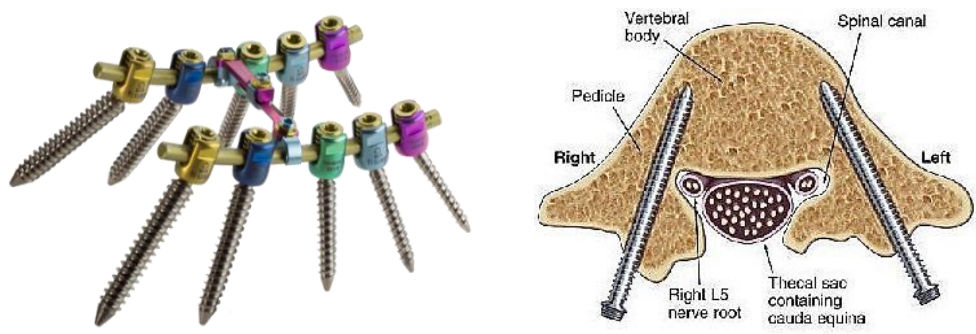

(a)
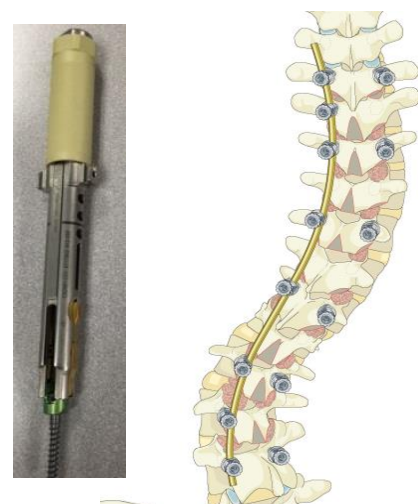

(b)

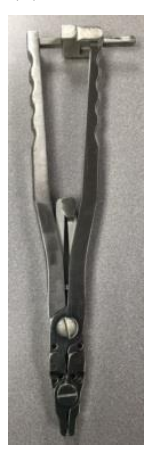

(c)

Figure 2. Technical aspects of surgical scoliosis correction. (a) Pedicle screw-anchored rod fixation: (left) assembly featuring inserted rods; (right) correct screw insertion into vertebral pedicles and body [7] [8]. (b) Reduction procedure whereby a rod is inserted into the tops of the screws; inset: reduction tower for rod insertion [4]. (c) 90-degree rotation that performs the correction; inset: pliers used for rod rotation [4]. Figures $b$ and c reproduced with permission; copyright by AO Foundation, Switzerland (4).

These limitations of state-of-the-art surgery simulation and planning have two main root causes. First, spinal ligaments are not easily delineated in MRI or CT: these tissues exhibit little contrast in relation to other soft tissues nearby. Second, even if one were able to identify these tissues (through segmentation), these tissue blobs would need to be decomposed into elements (by volumetric meshing), such as tetrahedral or hexahedra: this multi-material volumetric meshing is not done adequately in the current state of the art, namely in a manner that produces high-fidelity patient-specific models.

Recently, research on the large-scale compliance of the spine has been published in the biomechanics literature [9]. However, in the absence of a digital atlas of spinal ligaments, biomechanicists have to resort to modeling ligaments as a set of one-dimensional rods whose anchor points are imposed by hand, as depicted in figure 3, the limitations of which are described in detail in the Methods section.

With the proposed anatomical modeling approach, founded on a deformable multisurface model fitted to an anatomist-drawn Computer-Aided Design (CAD) template, we have the means to produce patient-specific finite element studies for a number of applications in orthopedics, emphasizing in this project the estimation of corrective forces in scoliosis surgery. This anatomical modeling technique, which addresses both 


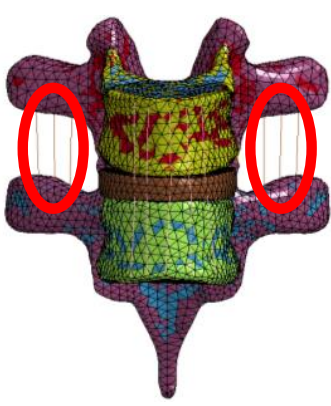

(a)

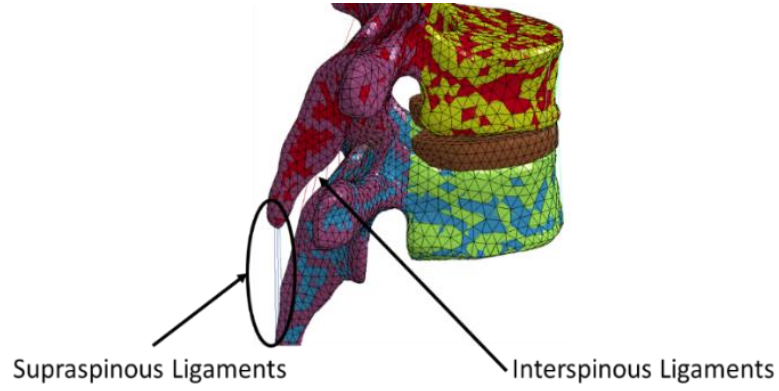

(b)

Figure 3. Motivation for an automatic ligamentous spine model computation: manual identification of ligamentous constraints for finite element studies (ongoing work). (a) Intratransverse ligaments (brown threads). (b) Supraspinous and interspinous ligaments (both labeled).

segmentation and meshing, warps the CAD-based anatomical template to any individual's CT/MR image dataset and can guide subsequent multi-tissue high-fidelity twostage tetrahedral meshing [10]. This two-stage tetrahedralization approach consists of i) a surfacic first stage, founded on a discrete deformable surface model [11], which produces a controlled-resolution high-fidelity triangulated boundary, followed by ii) volumetric second stage: controlled-resolution variational tetrahedral meshing [12] (found in CGAL [13]). The latter stage uses as input a prescribed triangulated mesh boundary resulting from the first stage. The deformable multi-surface model computation effectively integrates the segmentation and the first stage of the meshing in one step. For validation, we also planning some cadaveric image studies, featuring point clouds identified by an anatomist and coinciding with the boundaries of the ligaments.

\section{Methods}

\subsection{Anatomist-drawn Ligamentous CAD Model of the Spine}

The cornerstone of our approach to achieving descriptive personalized anatomical models of the spine is a CAD model that is commercially available through the TurboSquid website [14]. The justification for using such a model is that it is virtually impossible to volumetrically reconstruct the spinal ligaments from current routine imaging modalities, either CT or MRI-based, using voxel-based segmentation techniques. The descriptiveness required of the anatomy necessarily imposes a top-down, modelbased segmentation approach, which naturally maps to a multi-surface anatomical atlas.

Moreover, in existing efforts to run finite element studies of the ligamentous spine, while factoring in the constraining effect of the ligaments (figure 3), the current means of representing the ligaments is limited to a terse representation based on a set of linear constraints that are drawn by hand from one vertebral surface point to its opposing surface landmark: each ligament is thus approximated as a simple collection of 1D springs or stiff rods. We argue that, while this current approach is certainly an improvement over an entirely untethered spine model, it is potentially limiting in relation to the complexity of the $3 D$ ligament geometry and onerous in terms of user interaction, as is visible in figure $3 b$ in interspinous ligaments in particular. 


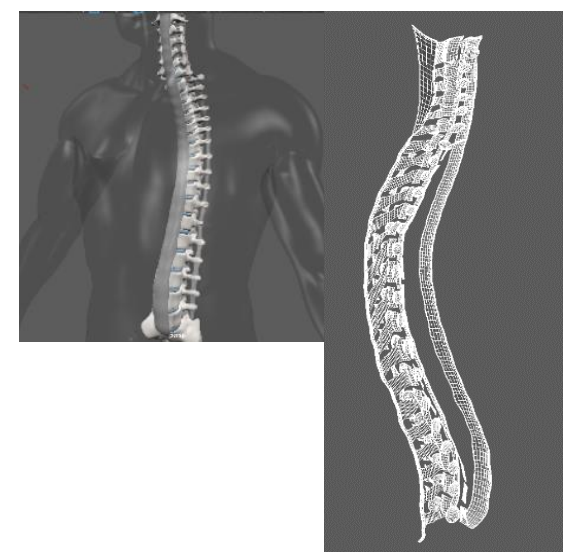

(a)

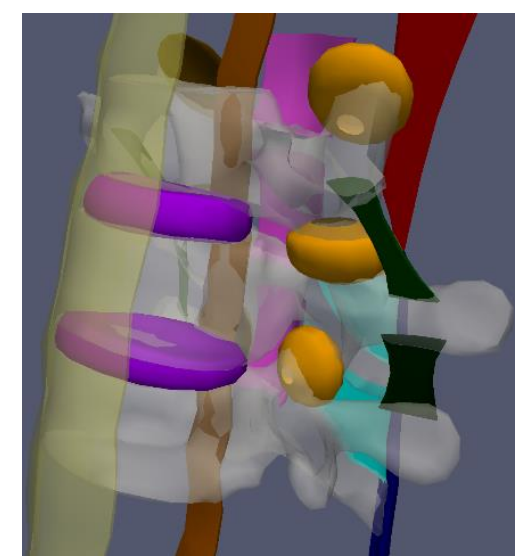

(b)

Fig. 4. Anatomist-drawn ligamentous CAD model of the spine. (a) Spine model displayed in its native Maya environment. Insert top left: surface rendering of the models of spline-based spine and ligament anatomical surfaces in Maya format, overlaid on translucent body surface. Right: wireframe rendering of the ligament model. (b) Close-up of a subset of the anatomy, converted to triangulated surfaces (stored in .obj format), featuring the following components as visualized with Paraview: C7, T1 and T2 vertebrae (white translucent), C7-T1 and T1-T2 IVDs (purple), local ligaments: intertransverse (dark green), interspinous (cyan), capsulary (orange), and ligamentum flavum (pink); ligaments spanning several vertebrae: anterior (yellow translucent) and posterior (rust) longitudinal ligaments, nuchal (dark red, spanning C1C6) and supraspinous (dark blue, spanning C7-sacrum) ligaments.

Moreover, should there be rheological studies published on spinal ligaments, which could be used to populate the material properties of such a finite element model of the spine, it would prove difficult to relate a small set of 1D springs to such properties acquired by stress-strain experiments on a shell-like structure. In contrast, these properties would naturally feed right into an anatomical model that faithfully replicates the curviplanar or volumetric structure, depending on the thickness of the ligament. In short, while manual rod/spring delineation is vital to tethering the spine, it under-represents the complexity of the constraining ligamentous geometry, which may have a dire impact on the fidelity of the finite element studies.

\subsection{Using Descriptive CAD Models for Segmentation - Deformable Multi- surface models}

As is true of all CAD drawings, the TurboSquid spine model (figure 4) is a collection of polygons, typically 2D B-spline quadrilateral patches, which can easily be converted to a triangulated surface: each quad patch is bisected into two triangles. Moreover, as can be seen in figure 5 and used extensively in our research, a triangulated surface can be used to initialize a deformable surface model, such as the Simplex [11], through geometric duality. The latter represents the second foundational aspect of our work:

\section{CAD B-spline surface $\rightarrow$ CAD triangulated surface} $(\rightarrow$ Watertight triangulated surface $) \rightarrow$ Deformable Simplex model

This recipe, although simple, is fundamental and surprisingly absent in the literature: 
this methodology suggests that we use an anatomist's CAD drawing of an arbitrary anatomy as a foundation for a deformable multi-surface model-based segmentation.

Although the results presented in this paper build on a naïve single-surface Simplex model, such as developed by Delingette [11], it is feasible to transition to a multi-surface approach [15] [16], which will be integrated into the second version of our methodology. The Simplex is a discrete deformable mesh model, characterized by a set of vertices linked by edges, and governed by a Newtonian model of vertex motion:

$$
m \frac{d^{2} \boldsymbol{P}_{i}}{d t^{2}}=-\gamma \frac{d \boldsymbol{P}_{i}}{d t}+\alpha \boldsymbol{F}_{\text {int }}+\beta \boldsymbol{F}_{\text {ext }}
$$

where $m$ and $\gamma$ represent vertex mass and damping, and the latter two terms are sums of internal and external forces. Moreover, an N-Simplex is a mesh where every vertex is linked to $N+1$ neighbors by edges. The 2-Simplex thus exhibits 3-connectivity, as shown in figure $5 \mathrm{a}$. Note the geometric duality between the 2-Simplex mesh in black and triangulated mesh in blue. The external force $\boldsymbol{F}_{\text {ext }}$ includes an image force that binds the model to anatomical boundaries of interest, typically characterized by strong gradient magnitude in a linear search space along the direction normal to each vertex.

There are two options for extending this single-surface deformable model to espouse multiple anatomical boundaries, and such an extension is foreseen in the near future. Gilles' multi-surface model featuring a static collision detection between individual surfaces [15]. Haq has integrated this multi-surface model with shape statistics force for spine applications [16]. Rashid's multi-surface Simplex model emphasizes shared boundaries based on multi-material surface extraction [17], which was used to achieve a lightweight deformable atlas of basal ganglia for efficient intraoperative-MRI-based guidance in robotic deep-brain stimulation. The latter multi-surface approach is advantageous in that it produces models of the weight-bearing anatomy with flush surfaces where needed, which is highly desirable in orthopedic finite elements applications.

It is worth noting that there are numerous resources available online to facilitate the computation of a watertight triangulated surface mesh, in the event that the surface model is derived from a voxel-based atlas by surface extraction (e.g. available on Paraview) or that there is a manifold inconsistency in the CAD model. Conversely, all it takes to throw off the geometric duality of the triangulated surface with the Simplex mesh is one hole or one non-manifold edge, whereby the deformable surface model is doomed to failure. Based on the recipe suggested in [18], Poisson Surface Reconstruc-

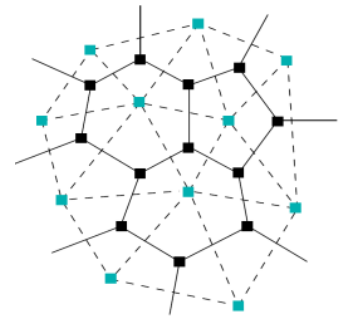

(a)

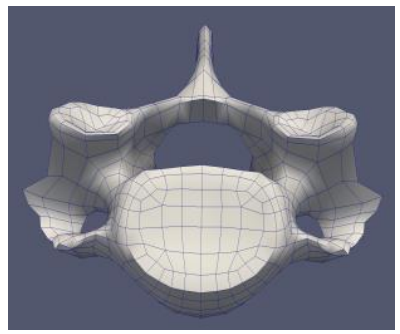

(b)

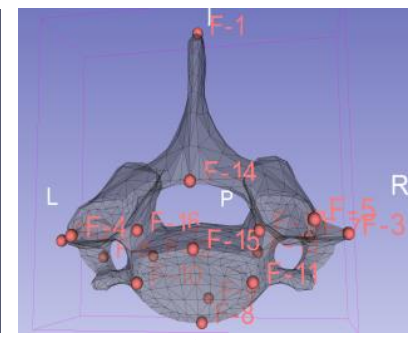

(c)

Figure 5. Computation of CAD-based Simplex vertebral model. (a) 2-Simplex surface mesh model in black, with dual triangulation in blue. (b) C7 vertebral CAD model, depicting Bspline quadrilaterals. (c) Triangulated surface, used to initialize Simplex by duality, with typical landmark configuration for initializing a vertebral registration by homologous point pairs. 
tion can be useful in correcting small surface inconsistencies (non-manifold edges, inconsistent surface normals, etc.). Furthermore, Quadric Edge Collapse decimation enables us to control surface mesh resolution while preserving topology. Both surface mesh processing algorithms are found in MeshLab [19]. In the case of the CAD model described above, most surface models were a watertight collection of quadrilaterals that could be diagonalized to produce a triangulated surface (through MeshLab's triangular mesh conversion). In the absence of a statistical pose model that can simplify the registration of each vertebra, we opted for a homologous point-based registration using 17 anatomical landmarks, identified using Slicer 3D planning software [20], which could reliably be located both on the vertebral model surface and in the target image. A few models, namely the capsular ligament, contained holes: sealing such openings in an unsupervised manner is the specialty of MeshFix [21].

In fact, for this simple proof of concept, the target image was taken from the SpineWeb database and featured segmented vertebrae, however it was still vital to warp the CAD model's vertebrae to the SpineWeb model. Our goal with this exercise is not to innovate on vertebral segmentation, but to demonstrate the merit of the chain effect whereby vertebral model transformations, which are unambiguously determined by sharp contrasts in a medical image, particularly CT, are aggregated and used to drive the registration of nearby soft-tissue structures. This is achieved while preserving the neighborhood topology throughout: ultimately, contiguity with unambiguously registered vertebral surfaces is exploited to constrain the positioning of soft tissue models.

The transformation used to initialize the Simplex warping is itself a simpler nonrigid mapping based on homologous point pairs, namely the Thin-Plate Spline (TPS) transformation [22]. This method leads to a gradient-based elastic transformation that maps a CAD vertebral surface model to the target boundary in the CT image, as can be seen from the overlay of warped CAD model surface over a surface extracted from the known boundary of the SpineWeb segmentation. Alternately, we can use an Iterative Closest Point method to establish the vertebral registration that will serve as anchor for the soft tissues, if we have a starting point of known anatomical boundaries of voxel-

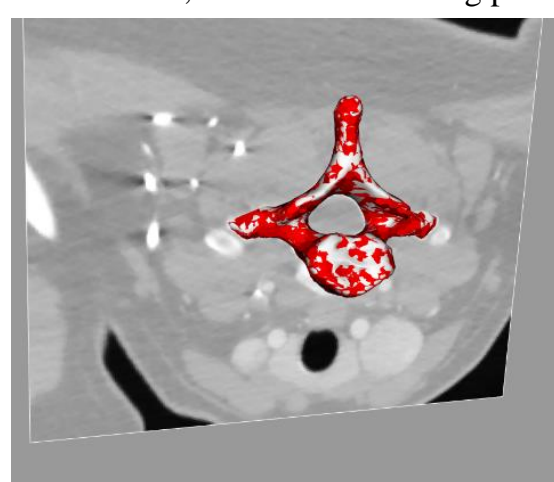

(a)

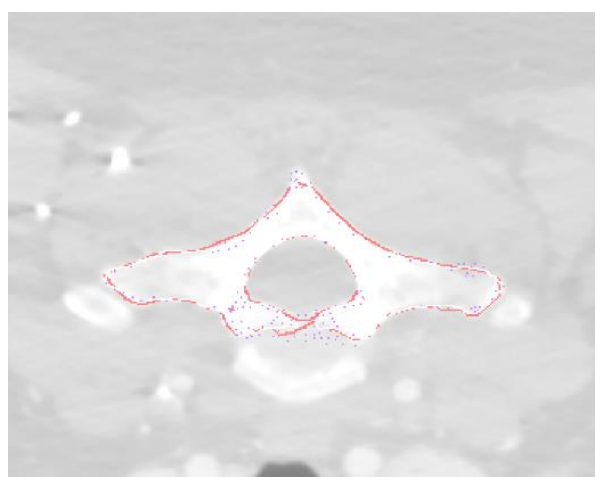

(b)

Figure 6. TPS-initialized, Simplex-mesh-based model-to-target image registration of vertebral CAD models. (a) Red warped model overlaid on white gold-standard surface from SpineWeb, with 3D-mapped 2D axial slice in background. (b) 2D contour depicting planar intersections of surfaces in a, mapped to red contour and purple gold-standard points respectively. 
based segmentation. Figure 6 depicts a typical result of a TPS-initialized vertebral surface model, after 600 iterations of the Simplex, overlaid in red on the known boundary of a SpineWeb model's C7 cervical vertebra, depicted in white. The set of such vertebral registrations will then serve to orient the soft-tissue surfaces attached to neighboring vertebrae. This vertebral registration is not the main aspect of the innovation: it serves as a foundational set of pitons we use to anchor our ascent, so to speak.

\subsection{Exploiting Well-defined Vertebral Transformations to Drive Ambiguous Ligament and IVD Model Registration}

The stable nonrigid registration of the $\mathrm{CAD}$ vertebral models can be aggregated to produce a putative registration of the IVD and ligaments, in a number of ways. One option would have been to distill each vertebral elastic registration into rotation and translation parameters that can be averaged or weighted according to proximity if dealing with a structure in between two vertebrae, and then use static collision detection to nudge overlapping surfaces away from each other. Ideally, if any image contrast information is available, through progress made with high-field MR scanners for example, softtissue-specific gradients could be used to finalize the image boundaries. Of course, one of the advantages of shared-surface boundary-preserving meshing is that vertebral surfaces will have direct implications for the boundaries of soft-tissue structures in contact with them. Alternately, as currently pursued here, a subsample of warped vertebral model points can be leveraged to determine a local TPS transformation that is in turn applied to a local neighborhood; where two warped vertebral models abut, they contribute to the local elastic transformation that is applied to soft-tissue surfaces affixed to them in the CAD model. Typical results are shown in the following section.

\section{$3 \quad$ Results and Discussion}

Currently, our validation is qualitative rather than quantitative, due to the difficulty of obtaining ground truth expert segmentations of the ligaments, which after all are elusive in existing CT or MRI alone. We can point to qualitative results that appear promising to the orthopedic surgeon on the team (senior author H.S.S.), as shown in figure 7. This figure depicts surface-rendered visualizations and rasterized images of planar intersections of the warped CAD model overlaid on the corresponding CT plane.

In fact, the planned validation strategy can briefly be described as follows. This validation methodology is currently under development through the efforts of the anatomists on the team (supervised by $3^{\text {rd }}$ author C.G.). As shown in figure 8 , the validation will exploit cadaveric imaging studies that integrate, on the one hand, high-resolution $\mathrm{CT}$ and MRI image acquisitions, interspersed with expert suturing of radiolucent thread at the surface of these ligaments, producing point cloud sets that will coincide with the boundaries, for comparison with those produced by the warped multi-surface model. These cadaveric studies could also lead to a shape statistics model, whose integration with multi-surface Simplex was demonstrated by Haq and Audette [16].

As mentioned above, this work is still in a nascent stage, but will soon integrate further surface meshing innovations. We could employ the static collision detection between neighboring surfaces to prevent overlap, as developed by Gilles [15] and also 


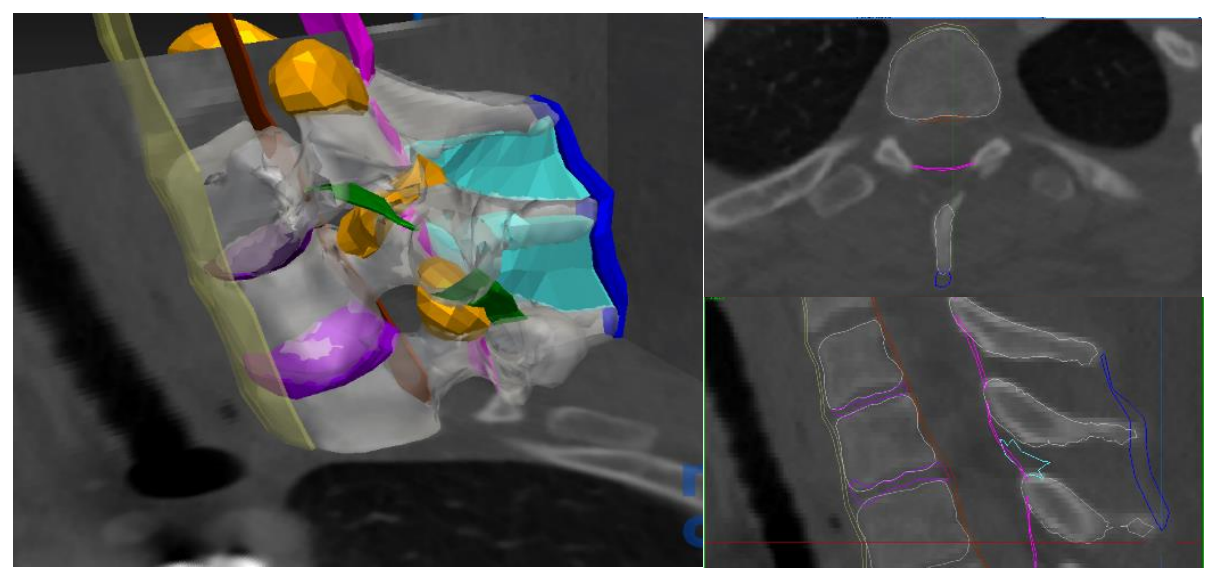

Figure 7. Left: MITK Workbench [23] scene with registered semitransparent vertebrae C7, T1 and T2, with two IVDs sandwiched between them and all ligament models of figure 4b, defined in relation to these vertebrae and rendered with the same color codes. Right top and bottom: axial and sagittal planes featuring colored contours coinciding with intersecting surface models. Right: 3D multi-planar view featuring embedded registered surfaces.

applied by Haq to the lumbar spine [16]. However, this implementation only prevents spatial overlap between two neighboring surfaces: it does not in any way ensure that these surfaces should be flush with one another. A better approach has been proposed by Rashid and Audette [17], as depicted in figure 9 below: multi-material surface extraction leads to a triangulated multi-surface complex with shared boundaries: this multi-surface model can then be converted to a deformable multi-surface Simplex model by geometric duality, which then allows us to warp the anatomy to the target image, while continuing to enforce shared boundaries. With a shared-boundary deformable multi-surface model, IVD-vertebra and ligament-vertebra interfaces will thus remain in perfect contact throughout the deformation.

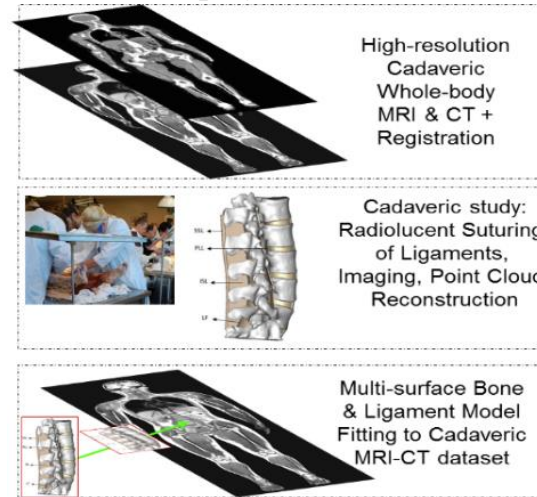

Figure 8. Planned validation study founded on cadaveric MR, CT and radiolucent suturing-based point cloud acquisition, coincident with ligament boundaries.

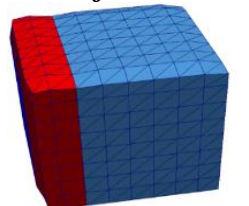

(a)

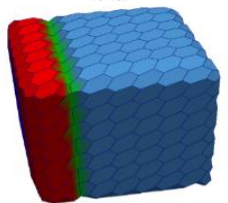

(c)

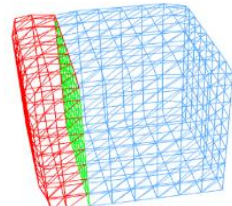

(b)

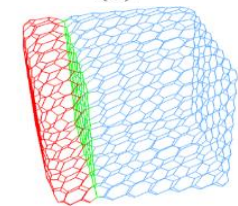

(d)
Figure 9. Planned multi-material sharedboundary multi-surface model. (a-b) Synthetic triangulated multi-material boundary, surface and wireframe-rendered. (c-d) Dual sharedboundary Simplex, (c) surface and (d) wireframe-rendered. Reproduced from [17]. 
Given that the starting point is a collection of individual surfaces, with tiny spaces between them, the implementation of a multi-material surface extraction will require a competitive Fast Marching method-based multi-front propagation of the various boundaries within a fine-resolution image volume, to flag overlaps and seal inter-surface "air pockets". Surfaces that should anatomically be in contact will then exhibit airtight contiguity in labeled fine-resolution image volumes. In turn, this airtight digital atlas of the ligamentous spine will be input to Rashid's shared-boundary-preserving multi-surface extraction. One aspect of this method that requires further work is a decimation technique for the multi-material Simplex model that preserves double vertices and edges on both sides of the interface. This decimation is needed to produce a suitably sparse multisurface complex that affords a robust, coarse-to-fine registration to the target image.

Last, the pre-Simplex TPS transformation exhibits undulations, which is typical of TPS interpolation of this nature. It is likely that a regularized approach, or alternately a thin plate-based approximation, will be less prone to such oscillatory behavior.

\section{Conclusions}

This paper presented a novel approach to producing personalized anatomical models of the spine that emphasize ligaments, in conjunction with the development of a finite elements simulation-based scoliosis surgery planning. While the meshing methodology is currently well proven, the application of anatomist-drawn CAD models is new and broadly applicable to orthopedic surgery planning in general: knee, shoulder, pelvis, and so on, provided that sufficiently descriptive and faithful CAD models of the relevant anatomy be available. This method also does not preclude further image-mediated refinements in the event that high-contrast delineation of the ligaments becomes available in MRI: the application of shared-boundary multi-surface Simplex models to tracking 3D image gradients has been demonstrated in deep brain data by Rashid [17].

\section{References}

1. Treatment of scoliosis. Correction and internal fixation by spine instrumentation. Harrington PR. 44-A:591-610. : Bone Joint Surg Am., 1962 Jun, J Bone Joint Surg Am, pp. 44-A:591-610.

2. Choosing fusion levels in adolescent idiopathic scoliosis. Trobisch PD, Ducoffe AR, Lonner BS, Errico TJ. 2013 Sep, J Am Acad Orthop Surg. , pp. 21(9):519-28. doi: 10.5435/JAAOS-21-09-519.

3. Multisurgeon assessment of surgical decision-making in adolescent idiopathic scoliosis: curve classification, operative approach, and fusion levels. Lenke LG, Betz RR, Haher TR, Lapp MA, Merola AA, Harms J, Shufflebarger HL. 2001 Nov 1, Spine (Phila Pa 1976). , pp. 26(21):2347-53.

4. AO Foundation. Adolescent Idiopathic Scoliosis Lenke 6 - Posterior Screws - With direct vertebral body derotation. [Online] www2.aofoundation.org.

5. The biomechanics of pedicle screw-based instrumentation. Cho $\mathrm{W}$, Cho $\mathrm{SK}, \mathrm{Wu}$ C. s.1. : J Bone Joint Surg Br., 2010 Aug;, Vols. 92(8):1061-5. doi: 10.1302/0301620X.92B8.24237. 
6. Pedicle Screw Fixation Under Non-Axial Loads: a Cadaveric Study. Bianco RJ, Aubin CE, Mac-Thiong JM, Wagnac E, Eng P, Arnoux PJ. s.l. : Spine (Phila Pa 1976), 2015 Oct 15., Vol. [Epub ahead of print].

7. Renovis . S100 Pedicle Screw System. [Online] http://www.renovissurgical.com/2011/09/s100-pedicle-screw-system/.

8. Neurology Update. Making Sure Pedicle Screws are Correctly Placed During Spine Surgery. [Online] https://mmcneuro.wordpress.com/2013/02/.

9. Quantitative comparison of ligament formulation and pre-strain in finite element analysis of the human lumbar spine. Hortin MS, Bowden AE. s.l. : Comput Methods Biomech Biomed Engin., 2016 Nov;, Vols. 19(14):1505-18. .

10. Topologically Faithful, Tissue-guided, Spatially Varying Meshing Strategy for Computing Patient-specific Head Models for Endoscopic Pituitary Surgery Simulation. Audette MA, et al. Jan. 2007, Journal of Computer Aided Surgery, pp. 12(1): 43-52.

11. General Object Reconstruction based on Simplex Meshes. Delingette H. 1999, Int. J. Comput. Vis., pp. 32(2):111-146 .

12. Variational tetrahedral meshing. . Alliez P, Cohen-Steiner D, Yvinec M, Desbrun M. 24, 3 (July 2005), 617-625., ACM Trans. Graph, pp. 24(3); 617-625; DOI=http://dx.doi.org/10.1145/1073204.1073238 .

13. CGAL. The Computational Geometry Algorithms Library. [Online] http://www.cgal.org/.

14. TurboSquid. TurboSquid 3D Spine Models . [Online] https://www.turbosquid.com/Search/3D-Models/spine.

15. Musculoskeletal MRI segmentation using multi-resolution simplex meshes with medial representations. Gilles B, Magnenat-Thalmann N. 2010 Jun, Med Image Anal., pp. 14(3):291-302. Epub 2010 Mar 1.

16. Statistical shape model construction of lumbar vertebrae and intervertebral discs in segmentation for discectomy surgery simulation. Haq R, Audette MA, et al,. Munich : Computational Methods and Clinical Applications for Spine Imaging, CSI 2015, LNCS 9402: 85-96., 2015. MICCAI 3rd Workshop on Computational Methods and Clinical Applications in Spine Imaging, pp. 85-96.

17. Deformable Multi-material 2-Simplex Surface Mesh for Intraoperative MRIready Surgery Planning and Simulation, with Deep-Brain Stimulation Applications. Rashid T, Audette MA, et al.,. Quebec City : BIVPCS 2017: Imaging for PatientCustomized Simulations and Systems for Point-of-Care Ultrasound pp 94-102, 2017.

18. Meshlab. Meshlab Stuff - Practical Mesh Processing Experiments. [Online] http://meshlabstuff.blogspot.com/2010/07/remeshing-and-texturing-1.html.

19. MeshLab. MeshLab. [Online] http://www.meshlab.net/.

20. Slicer 3D. Slicer 4.6 released. [Online] https://www.slicer.org/.

21. MeshFix. MeshFix SourceForge repository. [Online] https://sourceforge.net/projects/meshfix/.

22. Principal Warps: Thin-Plate Splines and the Decomposition of Deformations. . Bookstein, F. L. 1989., IEEE Trans. Pattern Anal. Mach. Intell. , pp. 11, 6 (June 1989), 567-585. DOI: https://doi.org/10.1109/34.24792.

23. MITK. Medical Imaging Interaction Toolkit (MITK) - Downloads. [Online] http://mitk.org/wiki/Downloads. 